\title{
Colonoscopia com Magnificação de Imagem: Análise da Variação Interobservadores para os Padrões de Criptas e Comparação das Imagens Endoscópicas com os Achados Histopatológicos
}

\author{
Magnification Colonoscopy Images: Analysis of Variation Interobservers for \\ Pit Patern and Comparison of Endoscopy And Histopatologic Findings
}

\author{
ESDRAS CAMARGOANDRADEZANONI ${ }^{1}$, RAULCUTAIT $^{1}$, MARCELO AVERBACH $^{1}$, \\ LIX ALFREDO REIS DE OLIVEIRA' ${ }^{1}$, CLÁUDIO ROLIM TEIXEIRA, PAULOALBERTOFALCO PIRES CORRÊA ${ }^{1}$, \\ JOSÉ LUIZPACCOS ${ }^{1}$, GIULIOF. ROSSINI ${ }^{1}$, LUIZH. CÂMARALOPES
}

${ }^{1}$ Membro titular da SBCP.

ZANONI ECA; CUTAIT R; AVERBACH M; OLIVEIRA LAR; TEIXEIRA CR; CORRÊA PAFP; PACCOS JL; ROSSINI GF; LOPES LHC. Colonoscopia com Magnificação de Imagem: Análise da Variação Interobservadores para os Padrões de Criptas e Comparação das Imagens Endoscópicas com os Achados Histopatológicos Rev bras Coloproct, 2006;26(4): 414-421.

RESUMO: A colonoscopia com magnificação de imagem (CMI) é considerada uma técnica que possibilita o diagnóstico diferencial entre lesões colorretais neoplásicas e não-neoplásicas. Este estudo avaliou a variação da concordância interobservadores para os padrões de criptas através de três endoscopistas experientes com a classificação de Kudo, e correlacionou esses aspectos morfológicos com os achados histopatológicos. Um total de 213 imagens de lesões magnificadas (polipóides e planas) forma coletadas de 161 pacientes consecutivos e apresentadas a três observadores independentes que expressaram opinião sobre o padrão de criptas predominante. Todas as lesões foram completamente excisadas e enviadas para estudo histopatológico. A estatística estimada de Kappa mostrou que o índice de concordância geral para padrões de criptas entre os três observadores foi bom (0,561). Com relação aos resultados histapatológicos, quando comparados aos padrões de criptas, observou-se: acuária de $84 \%$; sensibilidade de $91,4 \%$; especifidade de $67,2 \%$; valor preditivo positivo de $86,6 \%$ valor preditivo negativo de $79,3 \%$ e índice de Kappa de 0,61. Embora a reprodutilidade interobservadores dos padrões de criptas seja boa, a CMI não deve substituir o estudo histopatológico, pois não diferencia com a necessária segurança lesões neoplásicas de lesões não-neoplásicas.

Descritores: Colonoscopia; magnificação; neoplasia colorretal; padrão de criptas; classificação de Kudo.

\section{INTRODUÇÃO}

O CCR constitui-se em neoplasia frequente no mundo, tendo considerável participação na mortandade por câncer. A prevenção secundária para o CCR é altamente eficaz ${ }^{(1,2)}$ e possibilita a redução considerável em sua mortalidade para os indivíduos submetidos a rastreamento por colonoscopia por permitir a identificação e ressecção dos pólipos colorretais, lesões precursoras do câncer ${ }^{(3)}$.

Contudo, deve-se considerar a possibilidade da não-detecção de lesões polipóides ${ }^{(4,5,6)}$, a existência de lesões planas diminutas que apresentam comportamento biológico mais agressivo em relação as polipóides do mesmo tamanho ${ }^{(7,8,9,10)}$ ou, então, presença de carcinomas precoces plano-deprimidos que podem ser invasivos, mesmo quando muito pequenos $(11,12)$.

Com o advento dos videoendoscópicos de alta resolução, lesões planas e plano-deprimidas, consideradas um desafio ao diagnóstico por colonoscopia convencional, passaram a ser mais frequentemente detectadas $(13,14,15,16)$ e melhor definidas com o emprego da cromoendoscopia (CE) e colonoscopia com magnificação de imagem (CMI). Estas inovações permitiram também o diagnóstico diferencial endoscópico entre lesões neoplásicas e não-neoplásicas (11, 17,18,19,20,21,22).

O estabelecimento do diagnóstico histológico de uma lesão ainda durante a endoscopia poderia eco-

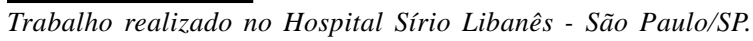

$\overline{\text { Recebido em 26/06/2006 }}$

Aceito para publicação em 14/07/2006 
nomizar tempo e recursos dispensados á ressecção e ao estudo histopatológico de lesões não-neoplasicas, bem como conduzir diretamente para o tratamento cirúrgico lesões neoplásicas que não são tratáveis endoscopicamente. Deve ser enfatizado também que as ressecções endoscópicas não estão isentas de complicações, como sangramento e perfuração ${ }^{(23,24,25)}$.

No presente, estudo pretendeu-se avaliar a classificação de Kudo ${ }^{(11)}$, empregando-se a experiência em CMI de três observadores independentes para se analisar a variação na interpretação dos padrões de criptas e, em especial, se a compatibilidade entre os diagnósticos endoscópicos e histapatológico autoriza ou não a sua aplicação na prática clínica.

\section{PACIENTES E MÉTODO}

Após obtenção do termo de consentimento informado, colonoscopia com cromoendoscopia foi realizada em 161 pacientes consecutivos, utilizando-se alta resolução (410.000 pixels) e magnificação de imagem - até 50x(Fujinon® EC410CM).

As colonoscopias foram realizadas indistintamente por cinco esperientes endoscopistas, todos com amplo conhecimento da técnica de magnificação de imagem, Duzentas e treze lesões colorretais foram categorizadas em polopóides ou planas e definidas quanto ao seu tamanho, localização e aspecto endoscópico, segundo a classificação macroscópica do carcinoma colorretal precoce, modificada por Kudo ${ }^{(11)}$ - (Figura 1).

Todas as lesões detectadas foram submetidas a jato de água frontal com a subsequente aplicação do agente mucolítico $\mathrm{N}$-acetilcisteína a $10 \%$. Procedia-se à cromoscopia de contraste com o corante índigo-carmim

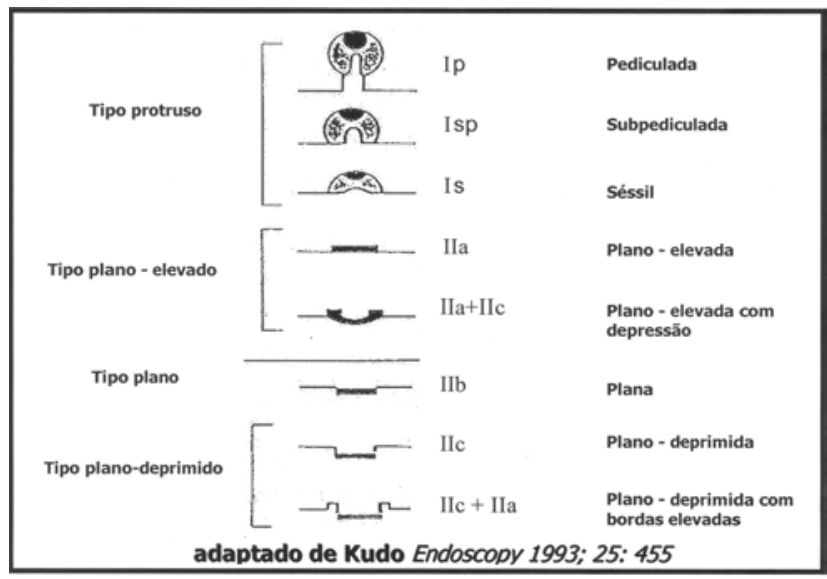

Figura 1 - Classificação macroscópica do câncer colorretal precoce (adaptado de Kudo, 1993). a $0,4 \%$ e quando necessário, ultilizava-se o corante violeta de cresil a fim de se obter avaliações mais detalhadas das características de superfície das lesões. Estas eram, então magnificadas e devidamente documentadas por fotografias (Olympus ${ }^{\circledR}$ OEP COLOR VIDEO PRINTER) que foram, posteriormente, apresentadas a três observadores independentes, de serviços distintos, com conhecida experiência em colonoscopia com magnificação de imagem, os quais fizeram, de maneira independente, suas análises dos diversos padrões de criptas, de acordo com a classificação proposta por Kudo (11) (Figura 2). Quando havia mais de um padrão de criptas em uma lesão, era solicitado aos observadores que considerassem o padrão mais agressivo.

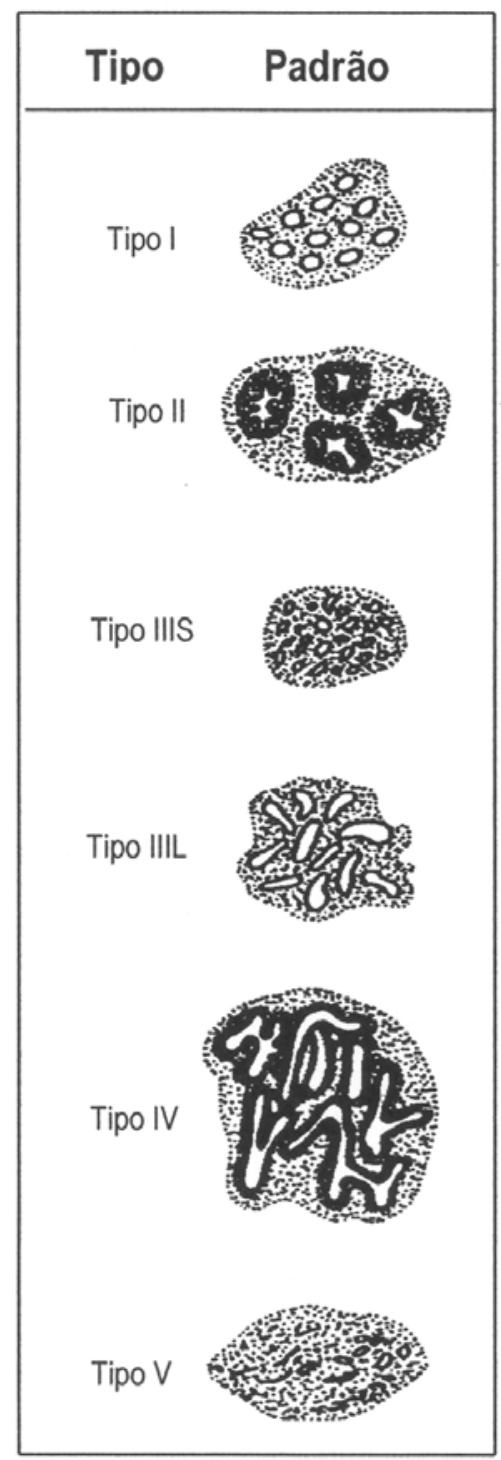

Figura 2 - Classificação dos padrões de criptas conforme Kudo (1993). 
Todas as lesões foram completamente excisadas e encaminhadas para estudo histopatológico. Os pólipos inflamatórios e os hiperplásicos foram classificados como lesões não-neoplásicas. Os adenomas (tubulares, túbulovilosos, vilosos e serrilhados), com componentes carcinomatoso ou não, foram designados como lesões neoplásicas. Para o presente estudo, lesões como lipomas, leiomiomas, tumores do estroma gastrointestinal e carcinóides foram consideradas também como não-neoplasicas, pois tratava-se de lesões extramucosas.

Os critéritos de exclusão foram os pacientes em investigação para polipose adenomatosa familiar e a documentação inadequada das imagens magnificadas, impossibilitando uma análise apropriada dos padrões de criptas.

Foi calculado o índice de concordância entre os três observadores para os diversos padrões de criptas e obtida a correlação do diagnóstico endoscópico com a diagnóstico histopatológico. Para tanto, consideraram-se como corretas as associações entre os padrões de criptas dos tipos I e II com a histologia nãoneoplasica e dos padrões de criptas dos tipos IIIS, IIIL, IV e V com a histologia neoplásica.

Os padrões de criptas encontra-se endoscopicamente demonstrados nas figuras 3 a 8 .

\section{RESULTADOS}

Dos 161 pacientes, 93 (57,8\%) eram homens e 68 (42,2\%), mulheres, com idade variando de 34 e 88 anos e média de 59,2 anos (desvio padrão de 11,9 anos).

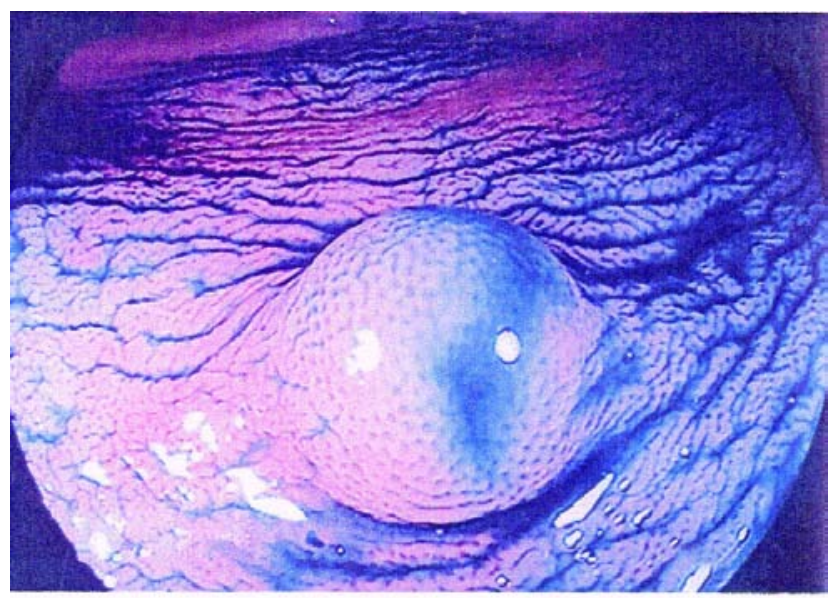

Figura 3 - Aspecto endoscópico de lesão polipóide após cromoendoscopia com índigo-carmim 0,4\% e magnificação de de imagem (50x) - Padrão de criptas tipo I

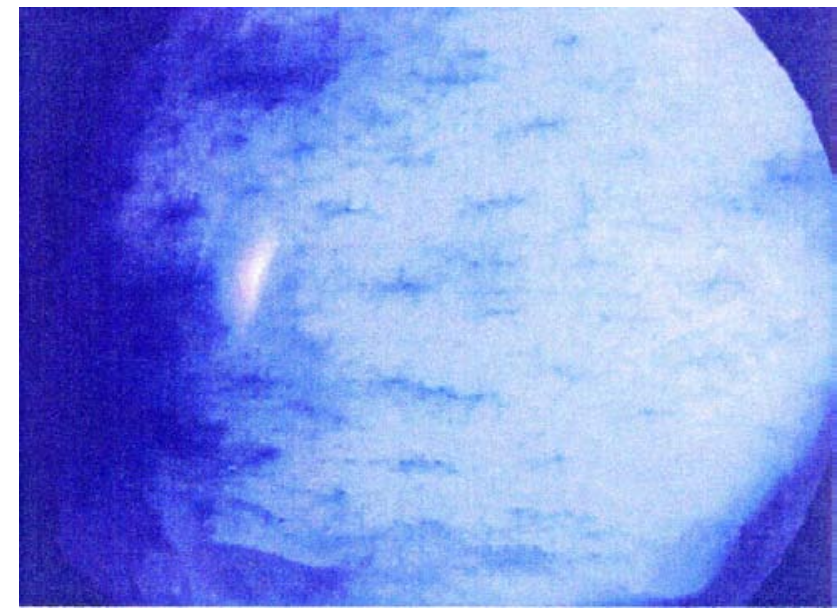

Figura 4 - Aspecto endoscópico de lesão polipóide após cromoendoscopia com índigo-carmim $0,4 \%$ e magnificação de imagem (50x) - Padrão de criptas tipo II.

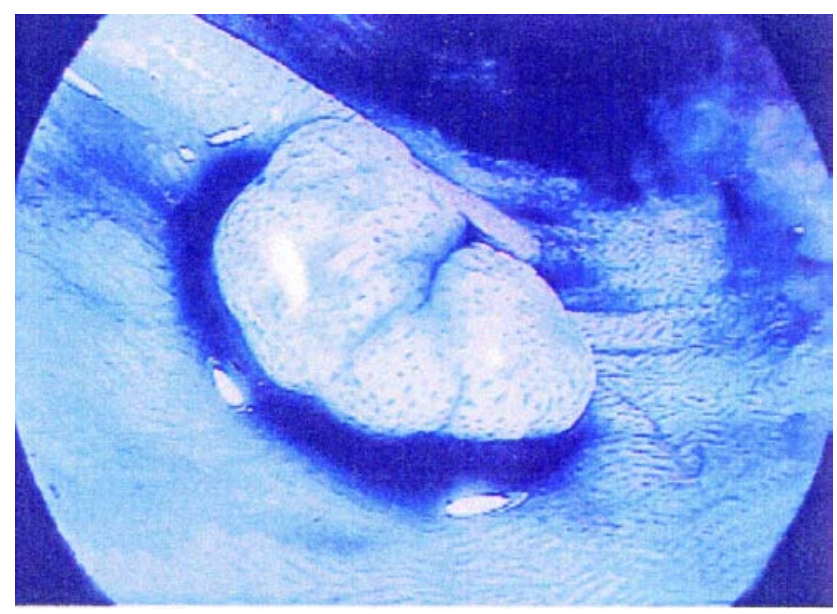

Figura 5 - Aspecto endoscópico de lesão polipóide após cromoendoscopia com índigo-carmim 0,4\% e magnificação de imagem (50x) - Padrão de criptas tipo IIIL.

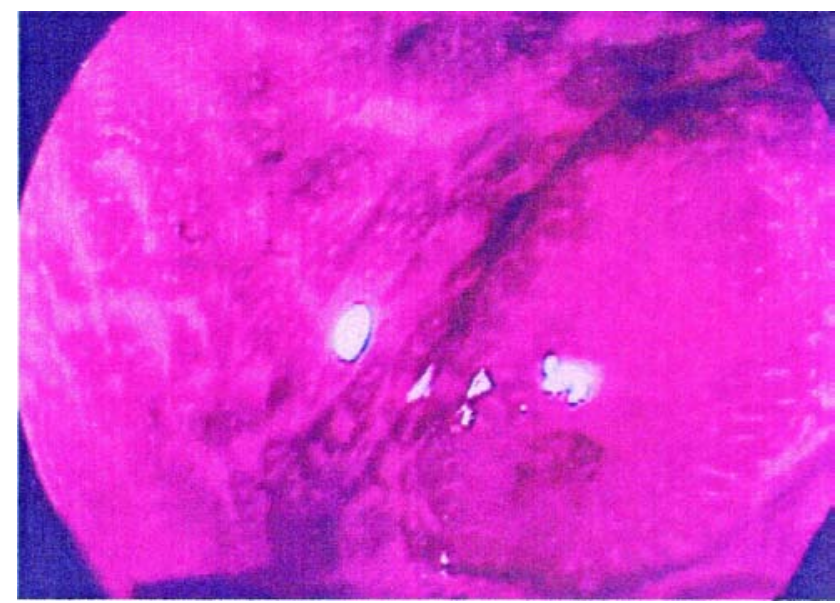

Figura 6 - Aspecto endoscópico de lesão plana após cromoendoscopia com violeta de cresil e magnificação de imagem (50x) - Padrão de criptas tipo IIIS. 

Esdras Camargo Andrade Zanoni e Cols.

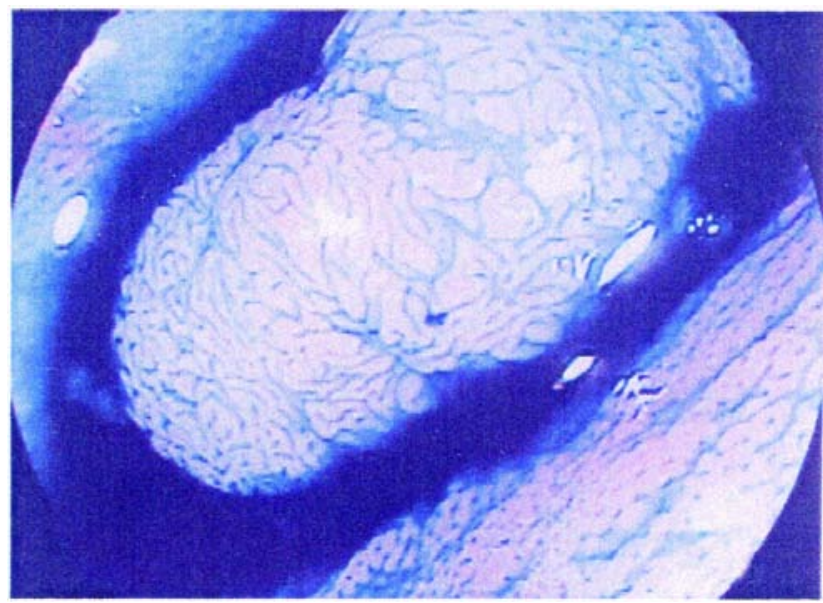

Figura 7 - Aspecto endoscópico de lesão após cromoendoscopia índio-carmin 0,4\% e magnificação de imagem (50x) - Padrão de criptas tipo $I V$.

Foram avaliados 213 lesões colorretais, sendo $191(89,7 \%)$ polipóides e $22(10,3 \%)$ planas, das quais $4(18,1 \%)$ era, lesões por espalhamento lateral (LST).

Conforme o estudo histopatológico, $134(70,2 \%)$ lesões polipóides eram neoplasicas e 57 (29,8\%)nãoneoplásicas. Com referência as lesões planas, 14 $(63,6 \%)$ eram neoplásicas e $8(36,4 \%)$ não-neoplásicas. O tamanho das lesões variou entre $0,1 \mathrm{~cm}$ e $2,4 \mathrm{~cm}$, sendo o tamanho médio das lesões de $0,6 \mathrm{~cm}$ (desvio padrão de 0,4 cm). Para as lesões não-neoplásicas, as localizações mais frequantes foram o reto e o sigmóide, enquanto que as lesões neoplásicas distribuíam-se quase que uniformemente no intestino grosso.

Foram estimados os coeficientes de concordância tipo Kappa, com seu respectivo intervalo de confiança de $95 \%$, para avaliar a concordância geral entre os três observadores, baseado nos padrões e criptas e após, para cada um dos seis tipos de padrões de criptas.

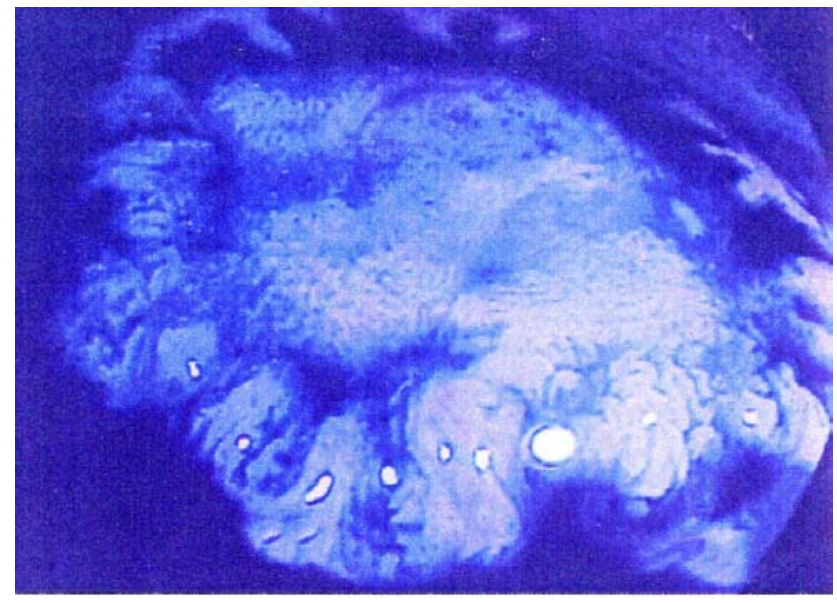

Figura 8 - Aspecto endoscópico de lesão plano-deprimida após cromoendoscopia com índigo-carmim 0,4\% e magnificação de imagem (50x) - Padrão de criptas tipo $V$.

Para a interpretação da estimativa do coeficiente, valores acima de 0,75 caracterizam excelente concordância, valores entre 0,40 e 0,75 mostram boa concordância e os abaixo de 0,40 mostram fraca concordância ${ }^{(26)}$.

$\mathrm{Na}$ avaliação da concordãncia geral entre os três observadores, com relação aos padrões de cripta, a estatística estimada de Kappa mostrou o índice de 0,561 (IC a 95\% [0,511 - 0,611]), indicando a rejeição da hipótese nula no nível de sgnificância de 0,05 (p?0,001), caracterizando a existência de concordância significativa entre os três observadores.

Na tabela 1 são apresentados os resultados referentes à avaliação do grau de concordância entre os observadores para cada padrão de criptas, de acordo com o critério definido acima.

Baseado nesses coeficientes, observa-se que existe boa concordância entre os observadores para os diversos padrões de criptas, exceto para o padrão do tipo V.

Tabela 1 - Análise do grau de concordância entre os observadores segundo padrão de criptas.

\begin{tabular}{lcccc}
\hline $\begin{array}{l}\text { Padrão de } \\
\text { Grau de criptas }\end{array}$ & Kappa & IC a 95\% & p-valor & concordância \\
\hline I & 0,525 & {$[0,448-0,603]$} & $<0,001$ & BOA \\
II & 0,584 & {$[0,507-0,662]$} & $<0,001$ & BOA \\
IV & 0,675 & {$[0,597-0,752]$} & $<0,001$ & BOA \\
V & 0,330 & {$[0,253-0,408]$} & $<0,001$ & FRACA \\
IIIL & 0,519 & {$[0,442-0,597]$} & $<0,001$ & BOA \\
IIIS & 0,428 & {$[0,351-0,506]$} & $<0,001$ & BOA \\
\hline
\end{tabular}


Rev bras Coloproct

Outubro/Dezembro, 2006
Colonoscopia com Magnificação de Imagem: Análise da Variação Interobservadores para os Padrões de Criptas e Comparação das Imagens Endoscópicas com os Achados Histopatológicos Esdras Camargo Andrade Zanoni e Cols.
Vol. 26 $\mathbf{N}^{\circ} 4$
Utilizou-se ainda o teste Qui-Quadrado de McNemar, onde os observadores 1, 2 e 3 foram comparados dois a dois, testando-se a hipótese de que apresentaram a mesma proporção de acerto. Comparando-se as probabilidades de acerto, foi observado que não houve diferença entre eles ( 1 e $2 / \mathrm{p}=0,121 ; 1$ e 3 / $\mathrm{p}=0,500$ e 2 e $3 / \mathrm{p}=0,405$, respectivamente).

Os resultados da correlação do diagnóstico endoscópico com o diagnóstico histopatológico para os três observadores estão representados nas tabelas 2,3 e 4 .

A tabela apresenta os índices de acurácia, sensibilidade, especificidade, valores preditivos positivo e nagativo, probabilidades de falso positivo e negativo e estatística de Kappa estimada para cada observador e sua médias.

\section{DISCUSSÃO}

O câncer colorretal (CCR) é a quarta neoplasia maligna mais diagnosticada no mundo e mais frequente do sistema digestótio. $\mathrm{O}$ grande impacto observado em relação à queda dos índices de mortalidade do CCR nas últimas décadas pode ser atribuído à utilização endoscópica de suas lesões precursoras ${ }^{(3,27)}$.

Os resultados provenientes do rastreamento do CCR certamente recomendam a colonoscopia como método de escolha para o diagnóstico de lesões colorretais. Porém, apesar de procedimento altamente sensível e específico para a detecção de neoplasias, não é teste perfeito e lesões adenomatosas pequenas podem passar despercebidas em até $27 \%{ }^{(5)}$. Diante disso, tem sido proposta a ultilização de técnicas de colocação da mucosa através de corantes (cromoendoscopia) e equipamentos endoscópicos de alta resolução e com magnificação de imagem (CMI). Com isso, tornou-se factível também a possibilidade de se predizer, com acurácia, o diagnóstico histológico de lesões colorretais polipóides e planas.

Porém, a literatura tem mostrado que os resultados referentes ao diagnóstico diferencial endoscópico entre lesões neoplásicas e não-neoplásicas, obtido pela CMI (100x), não tem sido confiáveis, principalmente para lesões não-neoplasicas, conforme mostram Togashi et al. (28) e Kato et al. ${ }^{(20)}$ que apresentaram acurácia, para as lesões não-neoplásicas, de 75,8\% e $75 \%$, respectivamente. Nas séries de Tung at al. ${ }^{(29)} \mathrm{e}$ Liu at al. ${ }^{(21)}$, foram obtidas especificidades de $64,6 \% \mathrm{e}$ $72,7 \%$, respectivamente. Dessa forma, lesões neoplásicas erroneamente diagnosticadas como, nãoneoplásicas, deixariam de ser excisadas.

Nesse estudo, para que o possível viés da não ultilização de imagens dinâmicas fosse anulado, as lesões magnificadas eram extensivamente observadas em sua plenitude, para que se obtivesse registro fotográfico adequado das características morfológicas e de superfície. O número de fotos para cada uma delas era variável a fim de que uma avaliação concreta e

Tabela 2 - Correlação do diagnóstico endoscópico x diagnóstico histopatológico para o observador 1.

\begin{tabular}{lccr}
\hline & \multicolumn{2}{c}{ Análise histopatológia } & \\
\cline { 2 - 3 } Observador 1 & Neoplásica & Não-neoplásica & \multicolumn{1}{c}{ Total } \\
\hline Neoplásica & 125 & 16 & $141(66,2 \%)$ \\
Não-neoplásica & 23 & 49 & $72(33,8 \%)$ \\
Total & $148(69,5 \%)$ & $65(30,5 \%)$ & $213(100,0 \%)$ \\
\hline
\end{tabular}

Tabela 3 - Correlação do diagnóstico endoscópico x diagnóstico histopatológico para o observador 2.

Análise histopatológia

\begin{tabular}{lccr}
\cline { 2 - 4 } Observador 2 & Neoplásica & Não-neoplásica & Total \\
\hline Neoplásica & 138 & 19 & $157(73,7 \%)$ \\
Não-neoplásica & 10 & 46 & $56(26,3 \%)$ \\
Total & $148(69,5 \%)$ & $65(30,5 \%)$ & $213(100,0 \%)$ \\
\hline
\end{tabular}


Rev bras Coloproct

Outubro/Dezembro, 2006
Colonoscopia com Magnificação de Imagem: Análise da Variação Interobservadores para os Padrões de Criptas e Comparação das Imagens Endoscópicas com os Achados Histopatológicos Esdras Camargo Andrade Zanoni e Cols.

Tabela 4 - Correlação do diagnóstico endoscópico x diagnóstico histopatológico para o observador 3.

\begin{tabular}{lrrrr}
\hline & \multicolumn{2}{c}{ Análise histopatológia } & \\
\cline { 2 - 3 } Observador 3 & Neoplásica & Não-neoplásica & Total \\
\hline Neoplásica & 143 & 29 & $172(80,8 \%)$ \\
Não-neoplásica & 5 & 36 & $41 \quad(19,2 \%)$ \\
Total & $148(69,5 \%)$ & $65(30,5 \%)$ & $213(100,0 \%)$ \\
\hline
\end{tabular}

não-duvidosa acerca do padrão de criptas fosse conseguida posteriormente pelos três observadores, o que foi atestado pelas coerência alcançada entre eles na interpretação dos padrões de criptas (Kappa - 0,561).

$\mathrm{Na}$ análise isolada para cada padrão de criptas, a concordância entre os três observadores foi também bastante aproximada, exceto quanto ao tipo $\mathrm{V}$ (0,330). Em estudo semelhante, Huang et al. ${ }^{(30)}$ obtiveram um índice estimado de Kappa de 0,716, mostrando excelente concordância entre os seis observadores selecionados. É possível que esse resultado tão expressivo tenha sido favorecido pela utilização de alta magnificação, que teoricamente pode aguçar a capacidade de interprretação para alguns padrões de criptas, principalmente os pequenos (IIIS e V).
Os resultados referentes à proporção de acerto entre os observadores mostram que não existem diferenças estaticamente significativas em relação às suas avaliações, indicando que a classificação de Kudo é bem interpretada e permite análise concisa e objetiva da arquitetura de superfície das lesões.

As médias dos resultados expostos na tabela 5 foram utilizadas como base para a discursão, uma vez que os intervalos de confiança (IC a 95\%) dos diversos índices se superpõem, o grau de concordância entre os três observadores é bom e não há diferenças em suas probabilidades de acerto. Analisando-se esses resultados, parece não haver dúvidas quanto à melhor efetividade do método frente às lesões neoplásicas (sensibilidade - $91,4 \%$; valor preditivo positivo - $86,6 \%$ ) e

Tabela 5 - Índices de Acuária, sensibilidade, especifidade, valores preditivos positivo e negativo, probabilidades de falso positivo e negativo e estatística de Kappa [IC a 95\%] para cada observador e suas médias.

\begin{tabular}{lcccc}
\hline Índice & Observador 1 & Observador 2 & Observador 3 & Média \\
\hline Sensibilidade & $84,5 \%$ & $93,2 \%$ & $96,6 \%$ & \\
& {$[77,8 \%-89,4 \%]$} & {$[88,8 \%-96,3 \%]$} & {$[92,3 \%-98,5 \%]$} & $91,4 \%$ \\
Especificidade & $75,4 \%$ & $70,8 \%$ & $55,4 \%$ & \\
& {$[63,7 \%-84,2 \%]$} & {$[58,8 \%-80,4 \%]$} & {$[43,3 \%-66,8 \%]$} & $67,2 \%$ \\
Valor preditivo + & $88,7 \%$ & $87,9 \%$ & $83,1 \%$ & \\
& {$[82,4 \%-92,9 \%]$} & {$[81,9 \%-92,1 \%]$} & {$[76,8 \%-88,0 \%]$} & $86,6 \%$ \\
Valor preditivo - & $68,1 \%$ & $82,1 \%$ & $87,8 \%$ & $79,3 \%$ \\
& {$[56,6 \%-77,7 \%]$} & {$[70,2 \%-90,0 \%]$} & {$[74,5 \%-94,7 \%]$} & \\
Acurácia & $81,7 \%$ & $86,4 \%$ & $84,0 \%$ & $84,0 \%$ \\
& {$[76,0 \%-86,3 \%]$} & {$[81,1 \%-90,4 \%]$} & {$[78,5 \%-88,3 \%]$} & $13,5 \%$ \\
Probalidade de Falso + & $11,3 \%$ & $12,1 \%$ & $16,9 \%$ & \\
& {$[7,1 \%-17,6 \%]$} & {$[7,9 \%-18,1 \%]$} & {$[12,0 \%-23,2 \%]$} & $13 \%$ \\
Probalidade de Falso - & $31,9 \%$ & $17,9 \%$ & $12,2 \%$ & $20,7 \%$ \\
Kappa & {$[22,3 \%-89,4 \%]$} & {$[10,0 \%-29,8 \%]$} & {$[5,3 \%-25,5 \%]$} & $2,5 \%$ \\
\end{tabular}

IC a $95 \%$ - intervalo de confiança a $95 \%$. 
quanto à maior dificuldade na caracterização das lesões não-neoplásicas (especifidade - 67,2\%, valor preditivo negativo - 79,3\%), conforme mostram algumas séries de CMI ${ }^{(20,21,28,29)}$. Vale enfatizar que os três observadores selecionados para esse estudo apresentavam curvas de aprendizado superior a 500 casos, aspecto ressaltado por alguns autores como fundamental ${ }^{(21,28,31)}$.

Embora a ultilização de alta magnificação possa significar melhores índices diagnósticos, o que se observa no presente estudo e deve ser ressaltado são seus resultados, que mesmo gerados à baixa magnificação, são superiores em alguns aspectos e no mínimo semelhantes em outros, aos resultados de algumas séries onde se empregou alta magnificação $(20,21,28,29,32)$. Deve-se questionar sobre a vantagem da ultilização de alta magnificação em relação à baixa magnificação.

Eisen et al. ${ }^{(33)}$, em estudo multicêntrico, ultilizando a colonoscopia de alta resolução com cromoscopia para diferenciar lesões neoplásicas de nãoneoplásicas, constataram que o índice isolado mais importante é o valor preditivo negativo, que necessita ser superior a $90 \%$ para se excluir confiavelmente um adenoma.

Extrapolando essa observação para o universo da CMI, é razoável admitir que a ocorrência de um valor preditivo negativo insatisfatório afeta o poder decisivo do método frente a condutas que podem acarretar o desenvolvimento do CCR.

Embora seja possível o diagnóstico diferencial endoscópico entre lesões neoplásicas e não-neoplásicas à luz da CMI, associada à CE, a análise dos padrões de criptas não deve substituir o estudo histopatológico, uma vez que não distingue com real segurança lesões neoplásicas daquelas inflamatórias ou hiperplásicas.

Talvez a CMI tenha como principal aplicação estabelecer o melhor tratamento (endoscópico ou cirurgico) para lesões neoplásicas plano-deprimidas, cujas detecções são um desafio na ausência de alta resolução, que ao contrário da CMI, mostra-se como tecnologia imprescindível.

\section{CONCLUSÃO}

Embora a reprodutibilidade interobservadores dos padrões de criptas entre endoscopistas experientes seja boa, a CMI não deve ser usada para se decidir quais lesões colorretais devem ser ressecadas ou não, uma vez que não consegue diferenciar com a necessária segurança lesões neoplásicas de não-neoplásicas.

Portanto, todas as lesões polipóides avaliadas pela CMI devem ser execisadas e submetidas a análise histopatológica.

ABSTRACT: Colonoscopy with magnified view is the most reliable nonbiopsy method for distinguishing non-neoplastic from neoplastic colorectal lesions. This study analysed lhe interobserver concordance in diagnosis of pit patem, with 3 experients endoscopists using Kudo Classification and correlating the this aspects with histological ones. A total of 213 magnificated images injuries (polipoides and plain) had been collected of 161 independent observing patients consecutive and presented tres that had expressed opinion on the predominant standard of criptas. All the injuries had been completely ressected and send for histopatológico study. The analysis esteem statistics of Kappa showed that the indice of general agreement for standards of criptas between the 3 observers was $84 \%$; sensitivity $91.4 \%$; specificity $67.2 \%$ positive preditivo value $86.6 \%$; negative preditivo value $79.3 \%$; e index of Kappa of $0,61 \%$. Although the interobserver reproduction of pit patern are good, the image for colonoscopia with magnificação, does not have to substitute the histopatologic study, therefore it does not differentiate with the necessary security neoplastic injuries of non neoplastic.

Key words: Magnification colonoscopy; neoplastic colorectal lesions; pit patem; Kudo classification.

\section{REFERÊNCIAS}

1. Hurlsotone DP, Fujii T, Lobo AJ. Erarly detection of colorectal cancer using high-magnification chromoscopic colonoscopy. Br J Surg 2002; 89:272-82.

2. Hawk ET, Limburg PJ, Viner JL. Epidemiology and prevention of colorectal cancer. Surg Clin North Am 2002; 82:905-41.
3. Winawer SJ, Zauber AG, Ho MN, O’Brien MJ, Gottlieb LS, Sternberg SS, et al. Prevention of colorectal cancer by colonoscopic polymectomy. The National Polyp Study Workgroup. N Engl J Med 1993; 329:1977-81.

4. Hixson LS, Fennerty MB, Sampliner RE, McGree D, Garewal H. Prospective study of the frequency and size distribution of polyps missed by colonoscopy. J Natl Cancer Inst 1990; 82:1769-72. 
5. Rex DK, Cutler CS, Lemmel GT, Rahmani EY, Clark DW, Helper DJ, et al Colonoscopic miss rates of adenomas determined by back-to-back colonoscopies. Gastroenterology 1997; 112:24-8.

6. Gorski TF, Rosen L, Riether R, Stasik J, Khubchandani I. Colorectal cancer after surveillance colonoscopy: false-negative examination or fast growth? Dis Colon Rectum 1999; 877-80.

7. Muto T, Kamiya J, Sawada T, Konishi F, Sugihara K, Kubota Y, et al. Small "flatadenoma" of the large bowel with special reference to its clinicopathologic features. Dis Colon Rectum 1985; 28:847-51.

8. Adachi M, Muto T, Okinaga K, Morioka Y. Clinicopathologic features of flat adenoma. Dis Colon Rectum 1991; 34:981-6.

9. Watanabe T, Sawada T, Kubota Y, Adachi M, Saito, Masaki T. et al. Malignant potential in flat elevations. Dis Colo $n$ Rectum. 1993;36:548-53.

10. Hurlstone DP, Cross SS, Adam I, Shorthouse AJ, Brow S, Sandres DS, et al. A prospective clinicopathological and endoscopic evaluation of flat and depressed colorectal lesions in the United Kingdom, Am J Gastroenterol 2003; 98:2543-9.

11. Kudo S. Endoscopic mucosal resection of flat and depressed types of early colorectal cancer. Endoscopy 1993; 25:455-61.

12. Teixeira CR, Tanaka S, Haruma K, Yoshiara M, Sumii K, Kajiyama G, et al. Flat-elevated colorectal exhibit a high malignat potential. Oncology 1996; 53:89-93.

13. Jaramillo E, Slezak P, Watanabe M, Rubio C. Endoscopic detection and complete removal oa a micro-invasive carcinoma present in a flat colonic adenoma. Gastrointest Endosc 1994; 40:369-71.

14. Treca A, Gai F, Di Lorenzo GP, Hreniuc H, Pasciuto A, Antoniellis F, et al. Conventional colonoscopy versus chromoendoscopy and magnifying endoscopy for the diagnosis of colorectal lesions: a comparative prospective study in 995 patients. Chir Ital 2004; 56: 31-6.

15. Hurlstone DP, Cross SS, Drew K, Shorthouse AJ, Brown S, et al. An evalution of colorectal endoscopic mucosal resection using high-magnification chromoscopic colonoscopy: a prospective study of 1000 colonoscopies. Endoscopy 2004; 36:491-98.

16. Kiesslich R, Neurath MF. Surveillance colonoscopy in ilcerative colitis: magnifying chromoendoscopy in the spotligth. Gut 2004; 53:165-7.

17. Tada M, Misaki F, Kawai K. A new approach to the observation of minute charges of the colonic mucosa by means of magnifying volonoscope, type CF-MB M (Olympus). Gastrointest Endosc 1978; 24: 146-7.

18. Kudo S, Hirota S, Nakajima T, Hosobe S, Kusaka H, Kobayashi $\mathrm{T}$, et al. Colorectal tumours and pit pattern. J Clin Pathol 1994; 47: 880-5.

19. Fujii T, Rembacken BJ, Dixon MF, Yoshida S, Axon AT. Flat adenomas in the United Kingdom: are treatable cancers beig missed? Endoscopy 1998; 30;437-43.
20. Kato S, Fujji T, Koba I, Sano Y, Fu KI, Parra-Blanco a, et al. Assessment of colorectal lesions using magnifyng colonoscopy and mucosal dye spraying: can significant lesions be distinguished? Endoscopy 2001; 33:306-10.

21. Liu H, Kudo S, Juch J. Pit pattern analysis by magnifying chromoendoscopy for the diagnosis of colorectal polyps. J Formos Med Assoc 2003; 102:178-82.

22. Bruno MJ. Magnofication endoscopy, high resolution endoscopy, and chromoscopy; towards a better optical diagnosis. Gut 2003; 52 (suppl IV):iv7-11.

23. Christie JP, Marrazzo $3^{\text {rd }}$ J. "Mini-perforation" of the colonnot all postpolypectomy perforations require laparotomy. Dis Colon Rectum 1991; 34:132-5.

24. Waye JD, Lewis BS, Yessayan S. Colonoscopy: a prospective report of complications J. Clin Gastroenterol 1992: 15:34751.

25. Waye JD, Kahn O, Auerbach ME. Complicatons of colonoscopy and flexible sigmoidoscopy. Gastrointest Endosc Clin N Am 1996; 6:343-77

26. Altman DG. Practical statistics for medical research. London: Chapman \& Hall; 1991. p. 611.

27. Trowbridge B, Burt RW. Colorectal Cancer Screening. Surg Clin North Am 2002; 82:943-57.

28. Togashi K, Konishi F, Ishizuka T, Sato T, Senba S, Kanazawa $\mathrm{K}$. Efficacy of magnifying endoscopy in the differential of neoplastic and non-neoplastic polyps of the large bowel. Dis Colon Rectum 1999; 42:1602-8.

29. Tung SY, Wu CS, Su My. Magnifying colonoscopy in differentiating neoplastic from nonneoplastic colorectal lesions. Am J Gastroenterol 2001; 96: 2628-32.

30. Huang Q, Fukami N, Kashida H, Takeuchi T, Kogure E, Kurahashi T, et al. Interobserver and intra-observer consistency in the endoscopic assessment of colonic pit patterns. Gastrointest Endosc 2004; 60:520-6

31. Kiesslich R, Jung M, DiSario JA, Galle PR, Neurath MF, Perspectives of Chromo and magnifying endoscopy? How, How muchm when, and whopm should we stain? J Clin Gastroenterol 2004; 38:7-13.

32. Coelho JCCGP, Savi D, Coelho JRGP, Araújo SEA, HabrGama A, Gama Rodrigues J. Assement of magnifying colonoscopy with chromoscopy in the differential diagnosis of neoplastic and non-neoplastic lesons of the large bowel. ABCD Arq Bras Cir Dig 2005; 18:64-70.

33. Eisen GM, Kim CY, Fleicher DE, Kozarek RA, Carr-Locke DL, Li TC, et al High resolution chromoendoscopy for classifiin colonic polyps: a multicenter study. Gastrointest Endosc 2002;55:687-94.

\section{Endereço para correspondência:} ESDRAS CAMARGO ANDRADE ZANONI

Rua Bruno Figueira, $369 / 11^{\circ}$ andar

Curitiba - Paraná 